\title{
A Case of Perianal Mucinous Adenocarcinoma Arising from an Anorectal Fistula Successfully Resected after Preoperative Radiotherapy
}

\author{
Ryo Ohta Koji Sekikawa Manabu Goto Kazuhiro Narita \\ Yasumasa Takahashi Hironari Ikeda Masataka Oneyama Yudai Hirata \\ Mikihiro Nakayama Yohta Shimoda Syun Sato \\ Department of Surgery, Institute of Gastroenterology, Kawasaki Saiwai Hospital, Kawasaki, \\ Japan
}

\section{Key Words}

Perianal mucinous adenocarcinoma - Anorectal fistula Preoperative radiotherapy

\begin{abstract}
A case of mucinous adenocarcinoma arising on a long-standing anorectal fistula is described. A 60-year-old man with a long history of mucinous discharge, pain and perianal induration underwent a biopsy of the external opening of the fistula that showed a mucinous infiltrating adenocarcinoma. Due to the large size of the tumor and the fact that it had extended into the surrounding tissue, preoperative radiotherapy was performed. Radiotherapy was given with $40 \mathrm{~Gy}$ in 25 fractions for 5 weeks through posterior and bilateral portals. After radiotherapy the tumor was markedly shrunk and the serum level of carcinoembryonic antigen was also improved. Abdominoperineal resection was performed 8 weeks after the termination of radiotherapy. Histological examination of the resected specimen revealed that the invasion of the tumor remained within the sphincter muscle and that no cancer cells were present on the surgical margin. The histological effect of radiotherapy was judged as grade $1 \mathrm{~b}$. This treatment can result in downstaging and R0 resection, which also has a possibility to prevent local recurrence. This case suggests that preoperative radiotherapy may play an important role in the definitive treatment of locally advanced perianal mucinous adenocarcinoma.
\end{abstract}


Ohta et al.: A Case of Perianal Mucinous Adenocarcinoma Arising from an Anorectal Fistula Successfully Resected after Preoperative Radiotherapy

\section{Introduction}

Perianal mucinous adenocarcinoma is a rare disease often associated with a longstanding anal fistula, representing approximately $2-3 \%$ of large bowel cancers [1]. The occurrence of a carcinoma in association with a fistula is probably due to chronic inflammation, although the rarity of the condition precludes any definite assumption in regard to the etiologic relationship of the fistula and carcinoma. In most patients, early diagnosis of this disease is difficult. Detection is usually late as the symptoms often initially mimic benign inflammatory conditions of the anorectal region and biopsies fail to reveal the infiltrating carcinoma. Therefore a tumor is often found in progress. Due to the rarity of this tumor and the lack of sufficient patients for controlled trials, there is no consensus regarding diagnosis and treatment strategies.

Surgical resection is generally accepted as the first choice of curative treatment. Abdominoperineal resection (APR) is advocated in the vast majority of patients to definitively remove the entire lesion and lessen the risk of local recurrence. Local resection is sometimes performed, but only in cases where the tumor margins can be confidently excised without damage to the anal sphincters. In order to prevent local recurrence, the APR has to be performed with wide local excision of both perianal soft tissue and the overlying skin. Recently locally advanced rectal cancer has been considered an indication for preoperative chemoradiotherapy (CRT). Preoperative CRT for rectal cancer has been shown to increase the probability of tumor resectability and to decrease the rate of local recurrence [2]. Radiotherapy alone or combined with chemotherapy can be a curative treatment for squamous cell carcinoma of the anal canal [3]. On the other hand, the role of radiotherapy has not been established for perianal mucinous carcinoma.

The aim of this article is to report a rare case of large perianal mucinous adenocarcinoma, arising from an anorectal fistula, successfully resected after preoperative radiotherapy.

\section{Case Report}

A 60-year-old man with a 45-year history of perianal fistula who had recently been suffering from mucinous discharge, pain and perianal induration was referred to hospital for treatment. Two years before, he had undergone surgery at another institution for incision and drainage of recurrent perianal abscess. Physical examination revealed an indurated, ulcerative lesion $7 \mathrm{~cm}$ in diameter with an external anal fistula opening (fig. 1a). The internal opening of the fistula was indicated at the right side of dentate line. There was no tumor in the rectum on digital examination. Histological examination of a biopsy specimen taken from the induration revealed mucinous adenocarcinoma. Magnetic resonance imaging (MRI) demonstrated a large demarcated tumor at the level of the anorectal junction with extension to the right side of the ischiorectal fossa and presacral space (fig. 1b). No enlarged pelvic lymph node was observed, nor was there evidence of distant metastasis. Neither colonoscopy nor barium enema showed any evidence of mucosal lesion in the colon or rectum. Laboratory data showed renal dysfunction caused by pyelonephritis, and the carcinoembryonic antigen serum level was extremely elevated to $40.8 \mathrm{ng} / \mathrm{ml}$ (normal $<5 \mathrm{ng} / \mathrm{ml}$ ).

To increase resectability and decrease the rate of local recurrence, preoperative radiotherapy was proposed without chemotherapy because of the renal dysfunction. Preoperative radiotherapy was given with 40 Gy in 25 fractions for 5 weeks through posterior and bilateral portals. After radiotherapy the tumor was markedly shrunk (fig. 1c, d) and carcinoembryonic antigen serum level gradually decreased to $13.0 \mathrm{ng} / \mathrm{ml}$. 
Ohta et al.: A Case of Perianal Mucinous Adenocarcinoma Arising from an Anorectal Fistula Successfully Resected after Preoperative Radiotherapy

8 weeks after radiotherapy the patient underwent radical resection. An APR with lymph node dissection was performed. The skin surrounding the tumor was excised with adequate tumor-free margin. The resected specimen revealed that the tumor had developed in the ischiorectal fossa, but there was no evidence of cancer extension to the mucosal surface of the anal canal and rectum (fig. 2). Histological examination of the excised specimen showed foci of mucinous adenocarcinoma involving the lower internal and external anal sphincter muscles, and the resected margins did not show any residual tumor (fig. 3). According to the UICC TNM staging criteria, the treatment outcome with preoperative radiotherapy revealed minimal response (grade 2). Metastases to the lymph node were not detected. After the operation, the patient died due to renal failure.

\section{Discussion}

Perianal mucinous adenocarcinomas arising from anorectal fistulae are rare tumors resulting in severe problems concerning diagnosis and treatment [4]. The pathogenesis, biological behavior and treatment of this disease remains controversial. It is suggested that adenocarcinoma developing in the perianal region is often associated with a long-standing anal fistula. Early diagnosis of a perianal tumor is difficult because of the absence of a tumor within the lumen of the bowel and the slow growth of a lesion hidden within the ischioanal fossa. Surgical resection is the first choice of curative treatment for perianal mucinous carcinoma [5]. As local excision is inadequate in most cases, APR with wide excision of both ischiorectal fossae and the overlying skin is recommended. On the other hand, radiotherapy alone or combined with chemotherapy can be a curative treatment for squamous cell carcinoma of the anal canal [6]. In the present case, total removal of the tumor without any residual tumor was quite difficult because of the size of the tumor and the fact that it had infiltrated the surrounding tissue.

Recently, preoperative CRT has become common practice for stage II and III rectal cancers. It has been well documented that neoadjuvant CRT induces tumor regression and downstaging, and therefore increases tumor resectability and R0 resection rate [2]. Therefore we performed preoperative radiotherapy to improve the resection rate and decrease the local recurrence rate. As the patient had renal dysfunction, additional chemotherapy treatment was not an option. After treatment the tumor was markedly shrunk and could be completely resected without skin grafting. This suggests that preoperative radiotherapy with or without chemotherapy is appropriate for treatment of perianal mucinous carcinoma arising from an anorectal fistula if adequate surgical margins cannot be ensured.

The role of CRT in the treatment of perianal mucinous adenocarcinoma has not yet been established. Unfortunately there are an insufficient number of reported cases undergoing this therapy to determine the prognosis for individual patients [7, 8]. Further tests are required to standardize the treatment of preoperative CRT for locally advanced perianal mucinous adenocarcinoma arising from an anorectal fistula.

In conclusion, perianal mucinous adenocarcinoma arising from an anorectal fistula is an uncommon malignant entity. If surgical treatment for anorectal fistula is unsuccessful in the long term, mucinous adenocarcinoma should be suspected. The result of this case suggests that preoperative radiotherapy appears to be a valuable alternative treatment for patients with perianal mucinous adenocarcinoma arising from an anorectal fistula. The advantage of this approach can result in downstaging and $\mathrm{R} 0$ resection, which prevents local recurrence. 


\section{Case Reports in
Gastroenterology}

\begin{tabular}{l|l}
\hline Case Rep Gastroenterol 2013;7:219-223 \\
\hline DOI: 10.1159/000351830 & $\begin{array}{l}\text { @ 2013 S. Karger AG, Basel } \\
\text { www.karger.com/crg }\end{array}$ \\
\hline
\end{tabular}

Ohta et al.: A Case of Perianal Mucinous Adenocarcinoma Arising from an Anorectal

Fistula Successfully Resected after Preoperative Radiotherapy

\section{References}

1 Salat A: Anal cancer. Eur Surg 2006;38:135-138.

-2 Kapiteijn E, Marijnen CA, Nagtegaal ID, et al; Dutch Colorectal Cancer Group: Preoperative radiotherapy combined with total mesorectal excision for resectable rectal cancer. N Engl J Med 2001;345:638-646.

-3 Nigro ND, Seydel HG, Considine B, et al: Combined preoperative radiation and chemotherapy for squamous cell carcinoma of the anal canal. Cancer 1983;51:1826-1829.

-4 Lee SH, Zucker M, Sato T: Primary adenocarcinoma of an anal gland with secondary perianal fistulas. Hum Pathol 1981;12:1034-1037.

5 Nelson RL, Prasad ML, Abcarian H: Anal carcinoma presenting as a perirectal abscess or fistula. Arch Surg 1985;120:632-635.

-6 Epidermoid anal cancer: results from the UKCCCR randomised trial of radiotherapy alone versus radiotherapy, 5-fluorouracil, and mitomycin. UKCCCR Anal Cancer Trial Working Party. UK Co-ordinating Committee on Cancer Research. Lancet 1996;348:1049-1054.

7 Gaertner WB, Hagerman GF, Finne CO, et al: Fistula-associated anal adenocarcinoma: good results with aggressive therapy. Dis Colon Rectum 2008;51:1061-1067.

8 Sierra EM, Saenz V, Martinez PH, et al: Mucinous adenocarcinoma associated with fistula in ano: report of a case. Tech Coloproctol 2006;10:51-53.
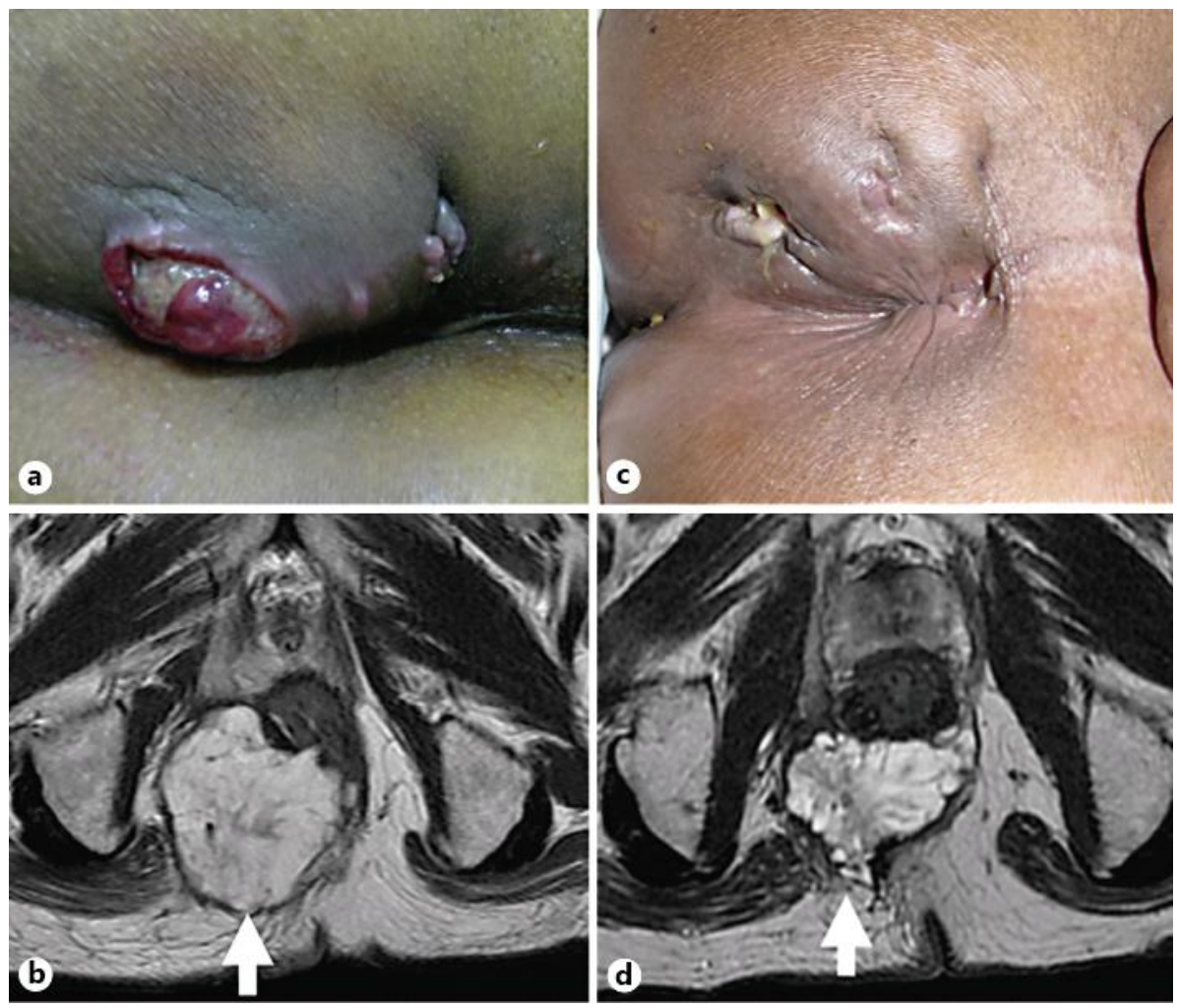

Fig. 1. Time series presentation of physical examination and MRI. a, b Assessment before start of preoperative radiotherapy. Initial physical examination revealed an indurated, ulcerative lesion $7 \mathrm{~cm}$ in diameter with an external anal fistula opening (arrow). Initial MRI demonstrated a large demarcated tumor at the level of the anorectal junction with extension to the right side of the ischiorectal fossa and presacral space. c, d Assessment at the end of preoperative radiotherapy. The tumor had shrunk remarkably and the patient had a good clinical response based on imaging (arrow). 

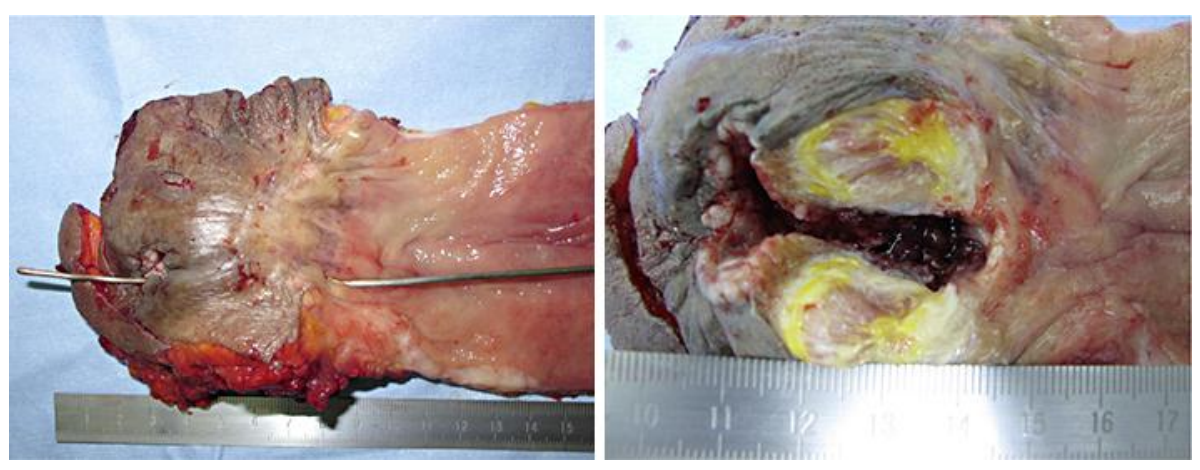

Fig. 2. The resected specimen revealed that the tumor had developed in the ischiorectal fossa, but there was no evidence of cancer extension to the mucosal surface of the anal canal and rectum.

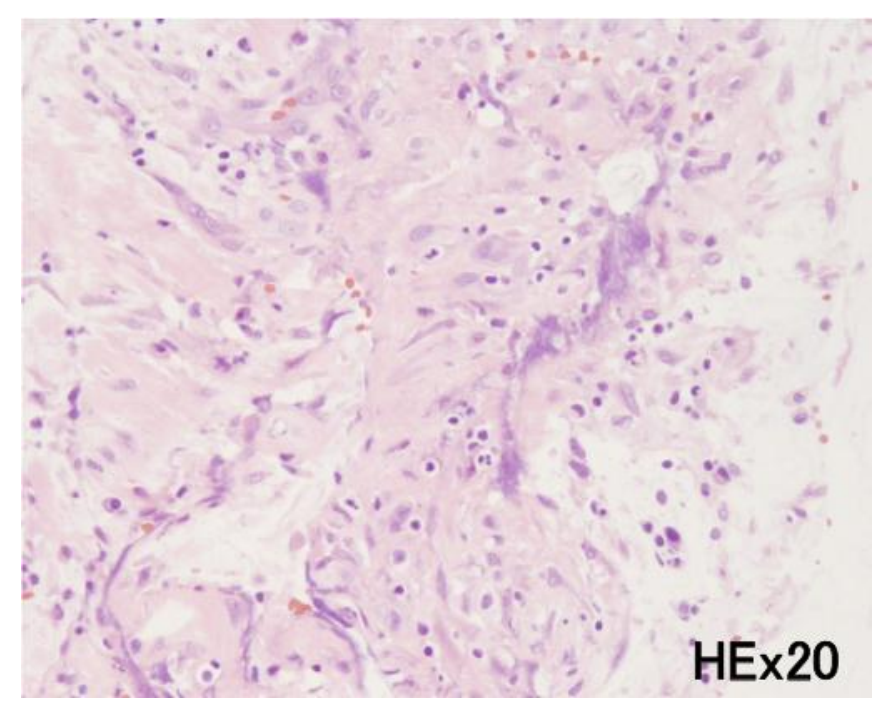

Fig. 3. Histological examination of the excised specimen showed foci of mucinous adenocarcinoma involving the lower internal and external anal sphincter muscles (H\&E stain, $\times 20)$. According to the UICC TNM staging criteria, the treatment effect with preoperative radiotherapy revealed minimal response (grade 2). 